Research Paper

\title{
Supervised learning methods for pathological arterial pulse wave differentiation: A SVM and neural networks approach
}

\author{
Joana S. Paivaa ${ }^{\mathrm{a}, \mathrm{b}}$, João Cardoso ${ }^{\mathrm{c}}$, Tânia Pereira ${ }^{\mathrm{c}, *}$

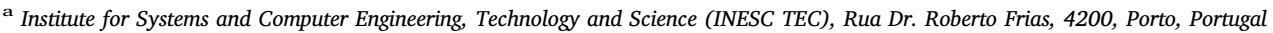 \\ b Physics and Astronomy Department, Sciences Faculty, University of Porto, Rua do Campo Alegre, 4169-007 Porto, Portugal \\ c LIBPhys-UC, Physics Department, University of Coimbra, Rua Larga, 3004-516 Coimbra, Portugal
}

\section{A R T I C L E I N F O}

\section{Keywords:}

Arterial pulse waveform

Morphologic features

Support vector machines

Neural network

Support vector machine recursive feature elimination

\begin{abstract}
A B S T R A C T
Objective: The main goal of this study was to develop an automatic method based on supervised learning methods, able to distinguish healthy from pathologic arterial pulse wave (APW), and those two from noisy waveforms (non-relevant segments of the signal), from the data acquired during a clinical examination with a novel optical system.

Materials and methods: The APW dataset analysed was composed by signals acquired in a clinical environment from a total of 213 subjects, including healthy volunteers and non-healthy patients. The signals were parameterised by means of 39 pulse features: morphologic, time domain statistics, cross-correlation features, wavelet features. Multiclass Support Vector Machine Recursive Feature Elimination (SVM RFE) method was used to select the most relevant features. A comparative study was performed in order to evaluate the performance of the two classifiers: Support Vector Machine (SVM) and Artificial Neural Network (ANN).

Results and discussion: SVM achieved a statistically significant better performance for this problem with an average accuracy of $0.9917 \pm 0.0024$ and a F-Measure of $0.9925 \pm 0.0019$, in comparison with ANN, which reached the values of $0.9847 \pm 0.0032$ and $0.9852 \pm 0.0031$ for Accuracy and F-Measure, respectively. A significant difference was observed between the performances obtained with SVM classifier using a different number of features from the original set available.

Conclusion: The comparison between SVM and NN allowed reassert the higher performance of SVM. The results obtained in this study showed the potential of the proposed method to differentiate those three important signal outcomes (healthy, pathologic and noise) and to reduce bias associated with clinical diagnosis of cardiovascular disease using APW.
\end{abstract}

\section{Introduction}

The blood pressure and related dynamic metrics have been extensively studied by the state-of-the-art literature and correlated with several diseases and population features [1-4]. Hemodynamically, rather than merely consider its maximum and minimum values, the overall shape of the arterial blood pressure waveform should be taken into account to describe the mechanical effects on the arterial wall and to give an adequate description of the arterial system behaviour. The studies of arterial pulse waveform opened a new vision about this biomedical signal and empathized the knowledge regarding the existence of linkages between cardiac and vascular dysfunction [5-8]. Information about the interaction between the left ventricle ejection and the physical properties of the arterial circulation can be determined by the descriptive and quantitative analysis of the arterial pulse pressure waveform [9]. Age related changes in aortic pressure waveforms exhibit early reflected waves and high systolic and pulse pressure amplitudes as a consequence of the arterial stiffness due to the early systolic wave reflections $[8,10]$. Four types of carotid pressure waveforms have been identified as suitable to determine the health condition and arterial stiffness degree based on the inflection point estimation and the backward waveform contribution for the systolic pressure. The clinical relevance of APW information boosted the development of several solutions for a non-invasive way to acquire such waveform [11]. A novel optical probe to assess the pulse pressure waveform was developed and tested in a clinical environment. This novel approach for the APW monitoring overcomes the main limitation of the existing types of measurements [11]. The current devices present some limitations due to the contact nature [11]. Emerging trends for APW measurement are moving away from more invasive technologies to non-

\footnotetext{
* Corresponding author.

E-mail address: taniapereira@lei.fis.uc.pt (T. Pereira).
} 
invasive and non-contact solutions. Optical sensors are an attractive instrumental solution due to their truly non-contact nature that allows the measurement of the skin surface pulsation at the carotid artery site for APW assessment [11]. This novel laboratory research prototype based on optical sensors boosted the knowledge of the APW and confirmed the correlation of arterial pulse waveform morphology-derived parameters with feature population and clinical conditions [12-16].

The current study is a follow-up of a preliminary work about the development of an automatic method that was able to distinguish valid data (part containing arterial pulse waveform) from non-relevant information (noisy waveforms) acquired by the referred optical system during a clinical examination, in order to reduce the variability between operators [17]. In this previous work a pool of 37 features split in different subsets was used based on the following types: amplitude features, time domain statistics, wavelet features, cross-correlation features and frequency domain statistics. A solution based on the combination between SVM RFE and other classifiers was implemented. Two classifiers were tested and compared: the k-nearest neighbours and SVM. The best result ( 0.952 accuracy) in the discrimination between signals and noise was obtained for the SVM classifier with an optimal feature subset. The features that have shown to be more relevant in the previous study were maintained in this work. In the present work it was intended to develop an automatic method to distinguish 3 classes of signal segments: healthy APWs, pathologic APWs and noisy parts (nonrelevant segments of the signal). An automatic method for APW classification introduces important features to the optical system for clinical usage: its ability to reduce the variability between operators and the possibility to decrease the clinical proceeding analysis duration that could be performed, in those conditions, by a less trained clinical professional, on the contrary of past scenarios. This multi-class problem is not completely new. In fact, some research teams have already proposed methods to differentiate between various types of APW using Machine Learning approaches [18-21]. However, as far as we know, this is the first time that a pattern recognition-based method able to differentiate "healthy" and "non-healthy" APW from "noisy" signal portions is proposed. By training a classifier to identify a class of "nonmeaningful" from other classes of "meaningful" signal portions could be an advantage, avoiding cases such for example the misclassification of a signal pulse that is just noise as a "non-healthy" sample, leading to a "false-positive" diagnosis. Thus, the purposed method could be an important contribution for helping physicians in the clinical diagnosis of cardiovascular diseases, by ensuring a less biased as possible cardiovascular risk assessment evaluation, similarly with several Machine Learning-based decision support approaches found in literature [22-24].

A sample database composed by both healthy volunteers and cardiovascular patients, in a total of 213 subjects, was used. A novel set of features was created, the multiclass SVM RFE method was used to select the most relevant features and a comparative study was performed to evaluate the performance of two classifiers, Support Vector Machine and Artificial Neural Networks. The solution based on the combination between SVM RFE and other classifier was already implemented with success in other biomedical challenges [25]. However, to the best of our knowledge is the first time that this combined approach (Multiclass SVM RFE + SVM or other classifier) is applied to the APW type's differentiation problem. Additionally, in general, it is not much common to apply the Multiclass SVM RFE method for feature selection. The binary SVM RFE is more frequently applied and in the context of binary problems [26]. SVM classifier was already used to distinguish between signals and noise parts in our previous study mentioned above [17]. ANN was also explored here and its performance was compared with SVM results for this novel APWs differentiation approach.

Neural Network (NN) algorithms have been frequently applied to solve data mining problems in biomedical applications [27-29], as for example to develop novel information extraction, diagnosis and clinical prediction techniques based on the electrocardiogram (ECG) signal
[30-35]. On the contrary, the application of NN algorithms in APWrelated problems is not being so frequently observed. However, OrjuelaCañon et al. [36] were able to develop a novel algorithm for accurate APW detection of fiducial points based on a Multilayer Perceptron Neural Network, a specific type of NN-based classification algorithm, and less expensive than the state-of-the-art techniques for being efficient without ECG-based additional information. Therefore, despite NN algorithms not being frequently applied for cardiovascular health assessment, several research teams have reported successful results for such applications $[36,37]$. Considering that this algorithm is suitable for fitting non-linear problems as the physiological ones, scientists have begun to apply NN-based and deep learning algorithms for abnormal health conditions detecting $[38,39]$.

The features that have shown to be more relevant in the previous study were maintained in this work. Additionally, morphology-based features were also used to introduce waveform structure information. For this reason, features based on inflection points, involving areas and intervals were introduced. This work contributes also as an improvement of the APW features study, in which techniques extensively used in other types of biosignals were applied, from which could result an interesting metrics toolset for APW analysis. Studies about APW have great relevance, contributing for enhancing the importance of this signal in clinical routines.

\section{Materials and methods}

\subsection{Data collection}

The segments used in this work were acquired using the referred optical system [40]. The APWs were acquired in a clinical environment from 213 subjects, including patients (68 subjects) and healthy volunteers (145 subjects), a population sample size that is consistent with several studies found in literature about cardiovascular risk assessment methodologies through pulse wave-derived metrics [41-45]. The study protocol was approved by the Ethical Committee of the Centro Hospitalar e Universitário de Coimbra (CHUC), Portugal. All the subjects were volunteers and agreed with the exposed terms by signing a written informed consent. Measurements were performed after a rest period of $15 \mathrm{~min}$ in the supine position at temperature-controlled environment. Each exam procedure consisted in the acquisition of a set of cardiac cycles at the carotid artery during few minutes, with the subject lying in supine position. Patient measurements were performed in a study population of 68 patients, with a cardiovascular pathology, who had undergone cardiac catheterization.

The dataset used is composed by APWs and noise, segmented into 1118 pieces (411 are labelled as normal APW, 124 are pathologic APW and 583 are noise). Ground-truth was defined by one highly trained physician with 10 years of work experience as "noise", "normal APW" and "pathologic APW". The physician is an experienced operator of this type of devices and trained to accurately identify and classify the data.

\subsection{Features study}

Some of the features that showed to be suitable in the previous study for identifying this type of physiological wave pattern were also included here. However, more focus has been done here to waveformrelated metrics, in order to extract features that reveal differences regarding the structure of a normal waveform and a pathological one. Eight time intervals and areas were therefore determined (Fig. 1). This kind of analysis, regarding the signal inflection points timings and areas, was extensively studied in the ECG or intracranial pressure pulse signals $[46,47]$.

Signal segments were parameterised by means of 39 pulse features divided in the following subsets: morphologic characteristics (systolic peak; reflection point; dicrotic notch; dicrotic peak and intervals and areas defined between them - Fig. 1) $[7,48,49]$; time domain statistics 


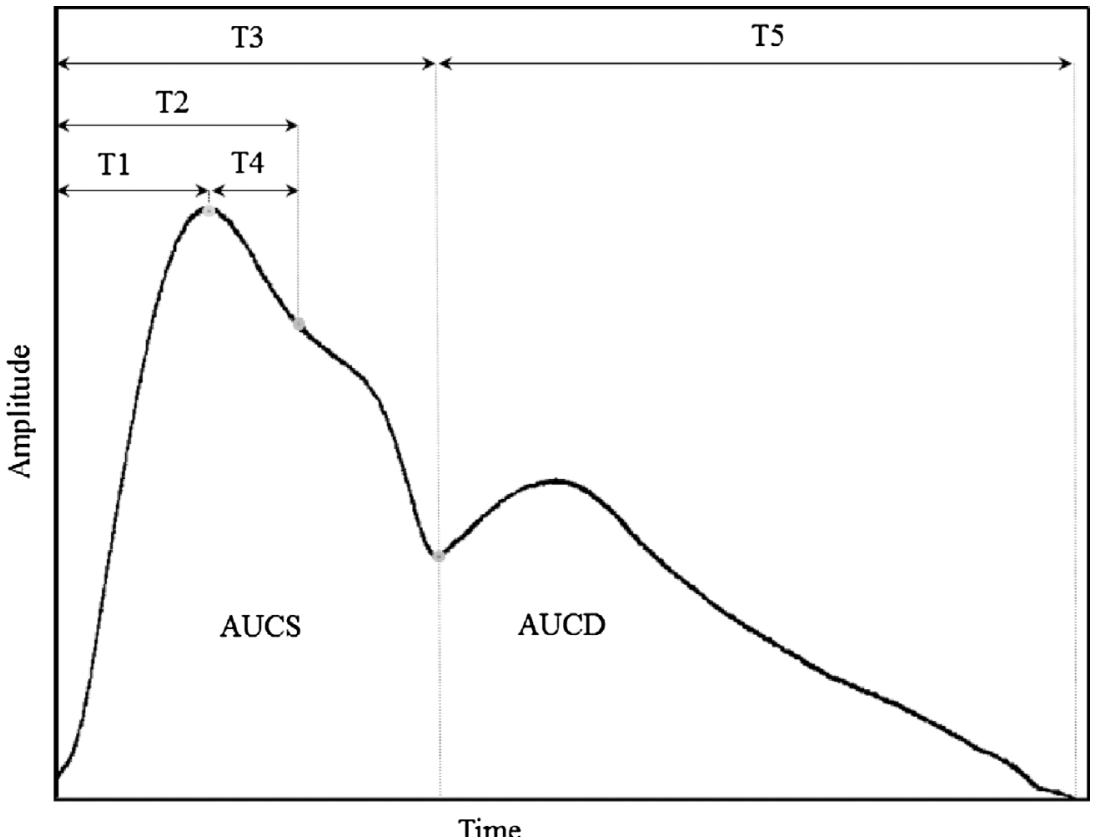

Fig. 1. Typical pressure waveform of a healthy subject and its inflection points, intervals and areas used here as APW features (T1 interval between the systolic peak, T2 -reflection point, T3 - dicrotic notch and T4 and T5 - foot of the wave and between them). The area under the curve was explored (AUC), and also determined for the systolic (AUCS) and diastolic (AUCD) period.
Table 1

Feature subsets and corresponding internal number identification used in the structure of the algorithm.

\begin{tabular}{lll}
\hline Group & Feature & $\begin{array}{c}\text { Feature } \\
\text { number }\end{array}$ \\
\hline $\begin{array}{c}\text { Morphologic } \\
\text { features }\end{array}$ & Time inflections point & $1-4$ \\
& Time intervals & $5-9$ \\
& Areas & $10-12$ \\
Time domain & Half Width & 13 \\
statistics & Mean, Median, Standard deviation, & $14-23$ \\
& Variance, Interquartile range, Range, & \\
Cross-correlation & Skewness, Kurtosis, Root mean square, & \\
features & Entropy & $24-27$ \\
Wavelet features & Haoss-correlation & $28-33$ \\
& Doubechies 4 & $34-39$ \\
\hline
\end{tabular}

(mean; median; standard deviation; variance; interquartile range; range; skewness; kurtosis; root mean square; entropy), cross-correlation features (maximum of cross-correlation with template waveform) [50], [51]; and wavelet features (relative power at six levels of wavelets for two mother wavelets, Haar and Daubechies (db4)) - Table 1 [7,48,49], [51-56].

For the amplitude features computation a low-pass filter (with a cutoff frequency of $30 \mathrm{~Hz}$ ) was used for noise filtering improving the signal differentiation, which is essential to detect waveform inflection points [12].

Due to the big difference in characteristics (amplitude and variation) of the feature components a normalization procedure was required. This task has a strong impact on the classification, and consists in subtracting to each value the mean over all training values and dividing it by the corresponding feature standard deviation [57].

\subsection{Features selection}

Feature selection allows the dimensionality reduction removing irrelevant and/or redundant features [58]. The issue of selecting an optimal set of relevant features plays an important role in pattern classification for ensuring the higher accuracy as possible $[59,60]$. In pattern recognition problems, high-dimensional feature sets impose a high computational cost and the risk of "overfitting". Feature selection addresses the dimensionality reduction problem by determining a subset of available features that is essential for classification and avoids the inclusion of redundant information, which could compromise classification performance, on the training model [58]. In this type of problems, it is desirable to discard features with no significant contribution, selecting a subset of features prior to design the subsequent classifier [61]. The implementation of feature selection method allows the identification of most relevant subset to distinguish the "healthy" vs. "non-healthy" or "noisy".

SVM RFE algorithm was used here to create a ranking score able to measure the significance of the initial set of features [62]. It starts with all of the original features set and removes one feature at a time step. Therefore, at each step, the feature ranking score sorted by order of significance in terms of the classification accuracy is generated according to the coefficients of the weight vector (W) of a linear SVM algorithm [62], [63]. According to the definition of SVM classifier, which uses a separating hyperplane with the maximal margin between two classes in order to distinguish one class from another, the best combination between the parameters kernel and $\mathrm{C}$ must be also screened for SVM RFE $[62,63]$. Usually, the kernel functions used to train SVMs are the linear and the Gaussian Radial Basis Function (RBF) [61]. Similarly to a standard machine learning problem, SVM-RFE parameters $\mathrm{C}$ and kernel type must be also optimized for each specific problem. In line with the SVM-RFE original proposed method procedure $[63,64]$, we considered a fixed kernel type, the linear kernel, for the SVM RFE algorithm. For reducing SVM-RFE evaluation process computational complexity and cost, we defined a previously limited range for the evaluated SVM-RFE C values. According to the results of our previous study [17], only very low values could ensure a good performance of the SVM RFE for APW types differentiation. Therefore, only the following $\mathrm{C}$ parameter values were assessed: $C=\left\{0.1,0.01,0.001,0.0001,1 e^{-5}, 1 e^{-6}, 5 e^{-7}, 1 e^{-7}, 1 e^{-8}\right\}$. Consequently, a different features ranking was generated for each one of these values.

However, SVM is a binary classifier [65], and therefore, in the case of multiclass problems, as the one that is presented here, it is necessary to convert a multiclass into a binary decision function $[66,67]$. The approach adopted here was based on the $\mathrm{R}$ software-based implementation of the SVM RFE algorithm that considers the division of the original multiclass classification problem into several binary classification problems $[64,68]$, based on the One-Against-One (OAO) 
procedure $[66,69]$.

Nine subsets from the features ranking were therefore generated using this SVM RFE implementation [69], one for each value of C tested in order to determine which SVM-RFE C value optimized final classification performance (the three signal types differentiation problem). The ranking of features obtained for each value of $\mathrm{C}$ is provided in Table 1, Supplementary Material (SM). Performance results of train and test sets were evaluated for each one of those rankings to identify the most suitable features sorting order.

\subsection{Classifiers}

The discrimination between APW waveforms and noise was already performed in a previous study [17], in which higher accuracy results were obtained using the SVM classifier with an optimal feature subset. Considering that the SVM classifier showed good performance results in that study, we decided to test here this same classifier against a FeedForward Back-propagation Neural Network [30,31]. Both SVM and NN algorithms were performed using the Statistical Pattern Recognition Toolbox [70] and Neural Network Toolbox, respectively, that run in Matlab .

SVM is able to cope with as aspects such as non-linearity and/or high-dimensionality of the input data, which are intrinsic attributes of physiological data, in particular, from the electrocardiographic and other cardiovascular-derived data. This property is particularly enhanced especially when SVM is used in combination with the Radial Basis Function (RBF) kernel function. Kernel functions are responsible to implicitly map non-linear features into a high-dimensional features space and use linear approaches for solving learning and estimation issues. This type of classifier has been widely used in several biomedical problems with great success [66,67], [71-75]. SVM is currently considered, for example, the most suitable type of classifier for ECG-based biometric applications $[65,76]$. Additionally, several authors have so far proved that SVMs are suitable to be embedded in low-cost wearable hardware modules for physiological monitoring [73,77-79].

As explained above, SVM is a binary classifier [66]. However, considering that the APW types differentiation problem that we are proposing to solve here is itself a multiclass problem, it was converted into several binary problems. However, on contrary of the SVM routine that was applied in the SVM RFE, the One-Against-All (OAA) strategy was used here, given the good results that are being reported using this approach in the scope of physiological data $[80,81]$. Also differently from the features selection procedure, the kernel function selected was based on the Radial Basis Function (RBF), once, despite being more complex, is able to separate nonlinear data, as the nature physiological data $[82,83]$. Therefore, the best combination between the parameters $\mathrm{C}$ and sigma was tuned in the performance evaluation phase (section 2.5).

Artificial Neural Networks are, similarly to SVM, supervised classification methods, being, as the name suggests, designed considering the working mode and information transmission adopted by our neurons $[66,84]$. The most important feature of this type of classification algorithm is its versatility and adjustment ability, being suitable to be applied to problems which cannot be described by a strict mathematical model, despite having a sufficiently representative set of samples [84].

A multi-layer neural network is therefore comprised by a large number of units (neurons) joined together, assuming a given connections pattern [84]. These units are divided into three types: input units or neurons, responsible for receiving the information to be processed; output neurons, at which are found the results of the processed data; and the hidden units or neurons, which consist in the elements that exist between the input and output neurons $[66,84,85]$. The performance of an ANN is dependent on several aspects: units activation functions; weight value of each input connection; the number of network hidden neurons used, etc. The determination of the best number of hidden neurons could be a problem associated with ANN-based
Table 2

Number of samples used per class in the training and test stages.

\begin{tabular}{|c|c|c|c|c|c|c|c|}
\hline \multicolumn{4}{|c|}{ Number of training samples } & \multicolumn{4}{|c|}{ Number of test samples } \\
\hline Class1 & Class 2 & Class 3 & Total & Class1 & Class 2 & Class 3 & Total \\
\hline 466 & 328 & 99 & 893 & 117 & 83 & 25 & 225 \\
\hline
\end{tabular}

classification issues $[66,84]$. This should not be too small, in order to avoid decision function poor approximation and generalization abilities; despite, on the contrary, a high number of hidden neurons could contribute for a higher complexity of the model, to overfitting events and a hard search for the global optimal solution $[66,84,86]$. For those reasons, the best number of ANN hidden neurons must be determined based on the information provided from the algorithm performance evaluation. Feed-forward Back-propagation ANN was implemented here using Matlab scripts available on the Neural Network Toolbox, and the number of hidden layers was optimized as described in section 2.5 .

\subsection{Performance evaluation}

Performance analysis was conducted considering both Accuracy (Acc) and F-Measure (F-M) values [66]. Considering that the dataset was unbalanced relatively to the number of samples per class (Table 2), performance evaluation was conducted also considering F-Measure values, beyond Accuracy. Considering that SVM-RFE C margin was also tuned using final classification performance values obtained in the three signal classes differentiation problem, possible data inhomogeneities in terms of number of samples per class were considered in the overall F-Measure results, Thus, classes imbalance was also indirectly corrected in the features selection method. The most suitable features ranking was selected taking only into account final F-Measure values obtained in the main classification problem (signal types differentiation).

The aim of the performance evaluation task is to find the classification model and associated parameters values that ensure the highest Accuracy and F-Measure, which here implied to find the best combination values between parameters C, SVM separating margin width, and sigma, the width for SVM gaussian kernel, for the SVM; and the number of hidden neurons/layers for the ANN. 5-folds Cross Validation (CV) method was used here for screening the best combination between classifier parameters, for both SVM and ANN [66].

In order to avoid overfitting and to test each model as closely as possible from a real scenario, $20 \%$ of the number dataset samples was randomly chosen for generating the test set (Table 2), which was never involved in the training phase. Therefore, the remaining $80 \%$ of the data was used for determining the best model parameters through the Cross-Validation method and to train the best classification model for both SVM and ANN, after being normalized in order to avoid withinsubject differences in amplitude and variation among features.

Training samples averaged values for each feature and corresponding standard deviation values were therefore stored in order to normalize novel test feature sets, being therefore possible to map novel values into the training model features space.

The following set of values for C and sigma were screened for SVM in the $C V$ method: $C=\{0.1,10,50,100,150,200\}$ and sigma $=\{0.01$, $0.1,1,10,100\}$. ANN best amount of hidden layers was also analysed in the CV considering the following set of values: net size $=\{3,5,10,15$, $20,30,50,100\}$. Considering that SVM RFE was previously used, the best features ranking from the ones that were generated by it, and the most suitable number of selected features $N$ were studied. Therefore, for each one of the nine rankings generated by the SVM RFE (Table 1, SM), the best combination between C and sigma regarding SVM and the best net size number for ANN were evaluated for each one of the following number of ranking features used for each classifier: $N=\{5,10,15,20$, 
Feature Ranking

$1 \mathrm{C}=0.1 \longrightarrow \begin{aligned} & 7262710824253935212019231112 \\ & 6313735162930363238912142215 \\ & 2834131743318\end{aligned}$

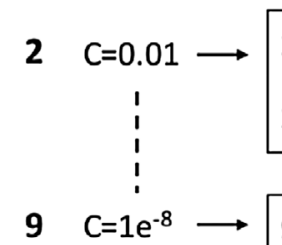

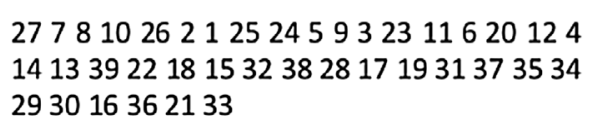

277810262125245932311620124 141339221815323828171931373534 293016362133

983427262672513232410163951 173218283420333111381929351437 213012153622

\section{Number of features used}

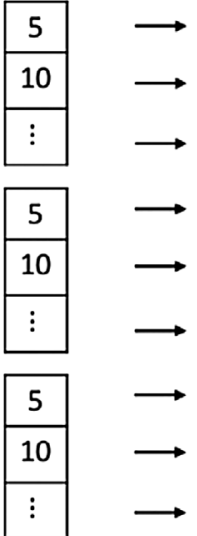

Best Parameters SVM ; ANN

$[\mathrm{C}$, sigma $]$; net size

$[\mathrm{C}$, sigma $]$; net size

$[\mathrm{C}$, sigma $]$; net size

[C, sigma] ; net size

[C, sigma]; net size

[C, sigma]; net size

[C, sigma]; net size

[C, sigma] ; net size

[C, sigma] ; net size

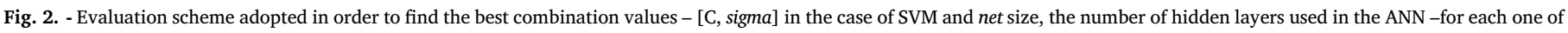
the ranking generated by the SVM RFE and each one of the number $N$ of features considered for the classification task.

25, 30, 35, 40, 45, 48\} (Fig. 2). According to this performance evaluation scheme, an optimal model (containing the best combination of classifier parameters values) for both SVM and ANN was determined, for each number $N$ of features used for a given features ranking from the nine that were generated by SVM RFE (Fig. 2).

Note that the best parameter classifier values were determined based on the results obtained in the Cross-Validation method and were used for testing each classifier, considering a set of "unseen" and "unknown" test samples.

The most suitable number of chosen features $N$ and combination of parameters values (C and sigma for SVM; and net size for ANN) were determined for each one of the two classifiers evaluated, SVM and ANN, for each one of the SVM RFE feature rankings. Statistical tests were also performed using the Statistics Toolbox from Matlab ${ }^{\circ}$, in order to identify the best classifier, SVM or ANN, as well as the best number of features and the best value for SVM RFE C, for each one of the classifiers evaluated (SVM and ANN). Parametric tests were used here since performance values follow a normal distribution, being ranged between 0 and 1. Student $t$-test was used in order to compare performance values between SVM and ANN. Kruskal Wallis tests were performed to determine the best value for C in the SVM RFE and the best value for $N$, number of features.

\section{Results and discussion}

The evolution of the F-Measure results corresponds to the combination of number of features $N$ and SVM RFE C values for both classifiers. Fig. 3 shows that higher performance values were obtained with SVM comparing to NN. The SVM presents a region with higher values of F-measure that corresponds to a range of combinations between SVM RFE C values and number of features, $N$. The F-Measure results obtained with NN had higher variability than with SVM (Fig. 3a, b).

Better results were obtained for SVM RFE features ranking $C$ values of 0.1 and 0.01 (Maximal F-M: 0.9957 for both conditions). However, considering also the results obtained in the training phase, (Table 2, SM), we conclude that the best SVM model is the one obtained considering a SVM RFE C value of 0.1 (Fig. 3c), and selecting the first 15 features from the correspondent features ranking (Fig. 3d). Indeed, the maximal training performance values were achieved for this SVM classifier: Maximal F-M: 0.9894; Maximal Acc: 0.9888, (Table 2 in SM). In general, performance results above 0.9700 were obtained regarding SVM for training and test phases, for both Accuracy and F-Measure, higher than the ones obtained in the previous study [17]. Regarding the number of selected features, $N$, only two values showed to be the best option across the different values for SVM RFE parameter C: 15 and 20
(Fig. 3a-c), being the first one the most frequently observed value. We could therefore conclude that the best value for the number of features selected $N$ regarding SVM classifier is 15 , which is reinforced by the statistical analysis performed (Table 3). A statistical significant difference was found between test F-M values, by varying the number of features $N$ ( $p$-value $<0.05$; Kruskal Wallis test; two-tailed), showing that the number of selected features significantly influences SVM performance. The 15 first features of the ranking correspondent to the best SVM model achieved were, in the majority, morphological-, cross-correlation- and time domain statistics-derived features. The less frequently selected type was related with wavelet decomposition, for being, probably, noisy and complex. The most frequently selected one is the morphological type, as in accordance with previous studies [18]. On the contrary of the number of features, the difference found between the performance values obtained for the several feature rankings (left side of Table 3) was not significant ( $p$-value $>0.05$; Kruskal Wallis test; two tailed), indicating that the order by which the 39 total features were selected did not significantly affected F-Measure, despite, as referred above, a better result was achieved using the feature ranking generated by $\mathrm{C}=0.1$.

Similarly to SVM, ANN showed Accuracy and F-Measure values above 0.9700 for both the train and test stages (Average F-Measure Test: $0.9852 \pm 0.0031$; Average Accuracy Test: $0.9847 \pm 0.0032$; Average F-Measure Train: $0.9852 \pm 0.0031$; Average Accuracy Train: $0.9848 \pm 0.0031)-$ Table 3 in SM. The best combination between SVM RFE C value and number of features $N$ selected was $5 \mathrm{e}^{-7}$ and 39 , respectively, achieving the ANN trained with those parameters the value of 0.9915 for test F-Measure. However, on contrary of SVM, the most suitable number of selected features $N$ is highly variable across the different feature rankings. Indeed, statistical results showed that the difference between test F-Measure across feature ranking $\mathrm{C}$ values considering different number of features was not significant ( $p$ value $>0.05$; Kruskal Wallis test) (Table 4). Therefore, it is not possible to determine the best number of features given the independency of test results relatively to this parameter. As observed above for SVM, the different order by which features were selected did not also significantly influenced the F-Measure test results for ANN classifier, Table 4 ( $p$-value $>0.05$; Kruskal Wallis). However, an observation that is common to both classifiers is their diminished F-Measure test values considering models trained with only 5 features, in comparison with other models. In fact, by performing several student $t$-tests comparing test F-Measure values for 5 versus all other number of features $N$, for each time, in a parwise fashion, it was observed that performance values were always significantly decreased for 5 features in comparison with any other number $N$ of features ( $p$-value $<0.05$; student $t$-test; two 

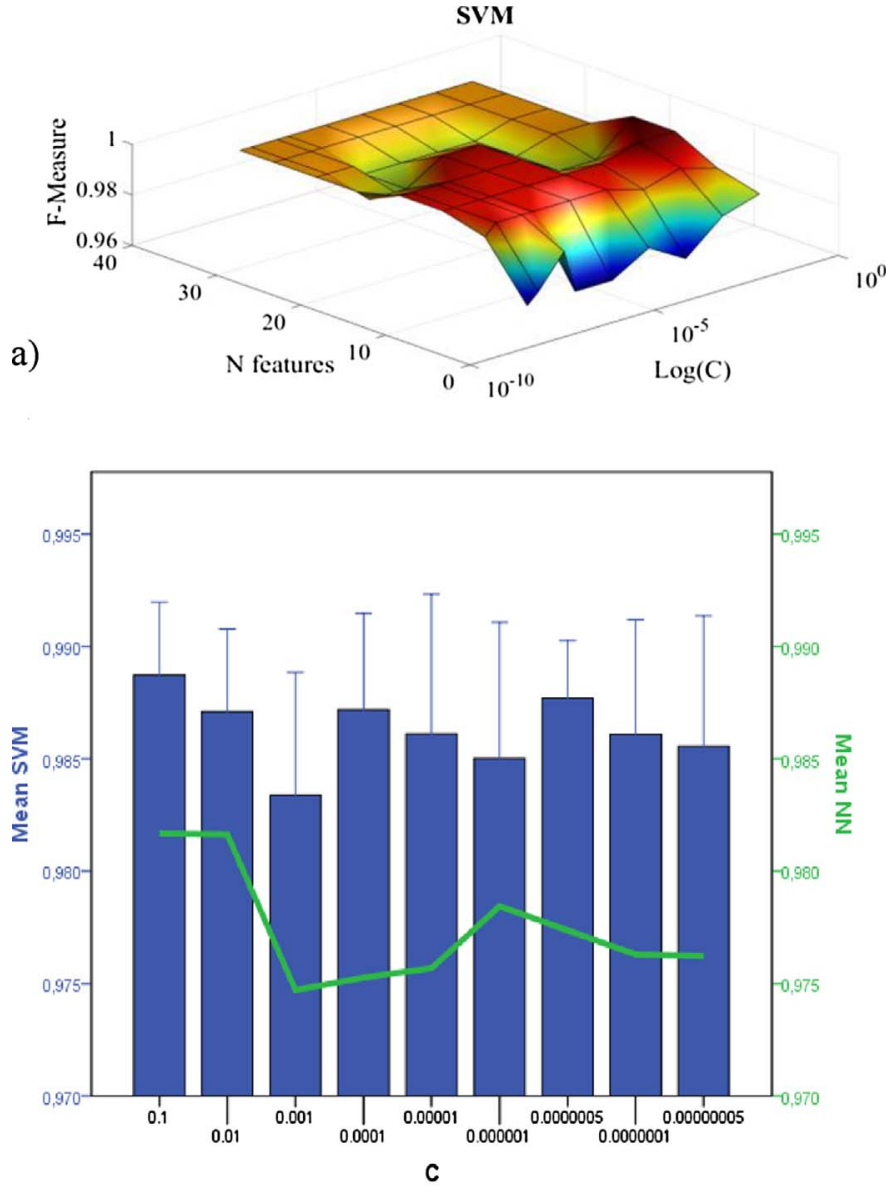

c)

Error Bars: +1-2. SE
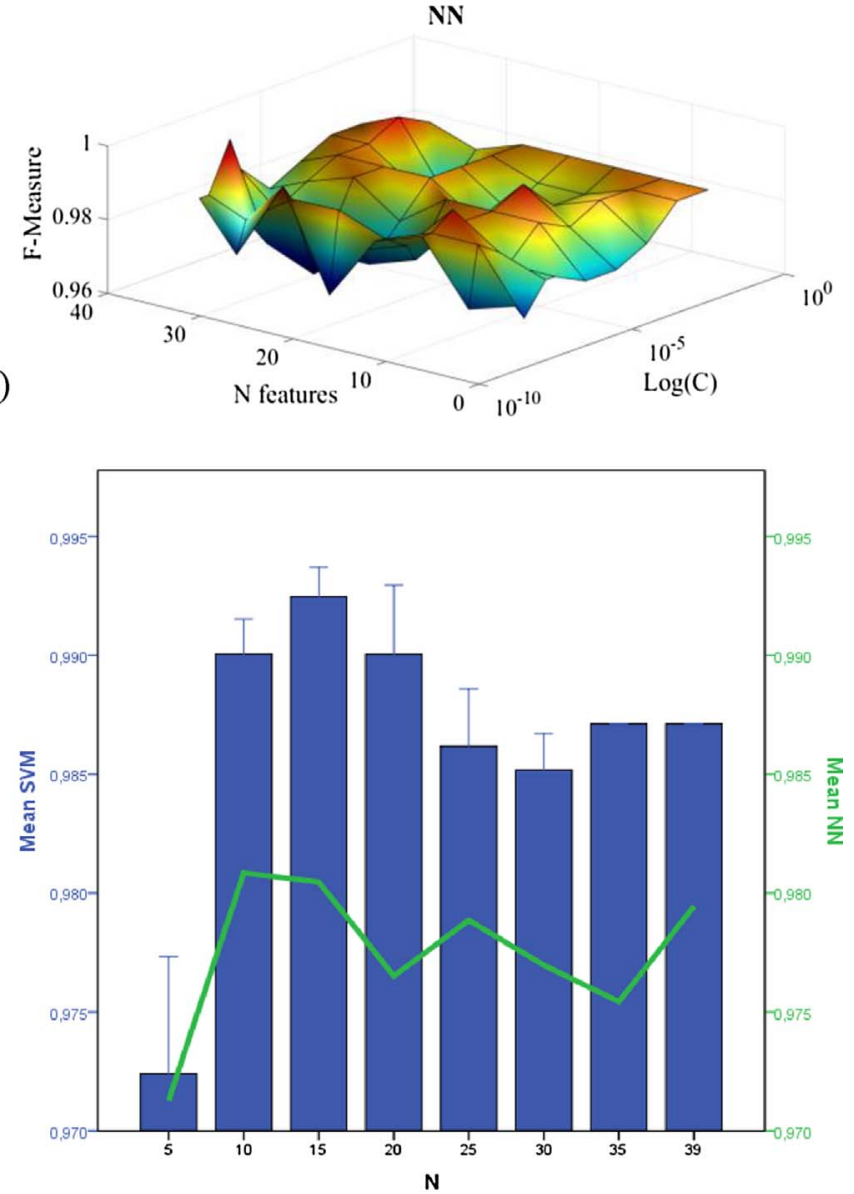

d)

Error Bars: +1- 2. SE

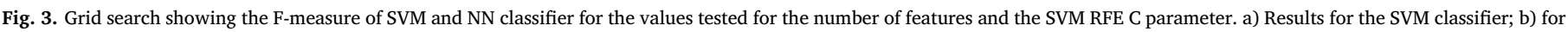
the NN classifier; c) and d) show the behaviour of the classifier performance with the different SVM RFE C and $N$ values, respectively.

Table 3

SVM average and correspondent standard deviation values for F-M obtained across number of features $N$ for each feature ranking value C (left); and across the different feature rankings for each number of selected features $N$ (right); and associated $p$-values for Kruskal Wallis tests (two-tailed; significance level: 0.05).

\begin{tabular}{|c|c|c|c|}
\hline \multicolumn{4}{|c|}{ Test F-M results Multiclass SVM } \\
\hline \multicolumn{2}{|c|}{ Feature ranking $C$ value } & \multicolumn{2}{|c|}{ Number of features, $N$} \\
\hline$C=0.1$ & $0.9887 \pm 0.0045$ & $N=5$ & $0.9724 \pm 0.0074$ \\
\hline$C=0.01$ & $0.9871 \pm 0.0052$ & $N=10$ & $0.9901 \pm 0.0022$ \\
\hline$C=0.001$ & $0.9834 \pm 0.0077$ & $N=15$ & $0.9925 \pm 0.0019$ \\
\hline$C=0.0001$ & $0.9872 \pm 0.0061$ & $N=20$ & $0.9900 \pm 0.0044$ \\
\hline$C=1 e^{-5}$ & $0.9861 \pm 0.0088$ & $N=25$ & $0.9862 \pm 0.0036$ \\
\hline$C=1 e^{-6}$ & $0.9850 \pm 0.0086$ & $N=30$ & $0.9852 \pm 0.0023$ \\
\hline$C=5 e^{-7}$ & $0.9877 \pm 0.0036$ & $N=35$ & $0.9871 \pm 0.0000$ \\
\hline$C=1 e^{-7}$ & $0.9861 \pm 0.0072$ & $N=39$ & $0.9871 \pm 0.0000$ \\
\hline$C=1 \mathrm{e}^{-8}$ & $0.9856 \pm 0.0082$ & & \\
\hline $\begin{array}{l}\text { p-value (Kruskal } \\
\text { Wallis) }\end{array}$ & 0.9306 & $\begin{array}{l}\text { p-value (Kruskal } \\
\text { Wallis) }\end{array}$ & $2.84 \mathrm{e}^{-8 *}$ \\
\hline
\end{tabular}

* Statistically significant (significance level: 0.05).

tailed), for both SVM and ANN. This suggests that it is relevant to train our model using more than 5 features.

By observing the results of the statistical comparison (Table 5), SVM classifier is significantly better in distinguishing the three type of classes, noisy, healthy and non-healthy (Test Average Acc SVM: $0.9917 \pm 0.0024$; Test Average Acc ANN: $0.9847 \pm 0.0032$; Test Average F-M SVM: $0.9925 \pm 0.0019$; Test Average F-M ANN: $0.9852 \pm 0.0031$ ), as it was expected, based on evidences reported in
Table 4

ANN average and correspondent standard deviation values for F-M obtained across number of features $N$ for each feature ranking value C (left); and across the different feature rankings for each number of selected features $N$ (right); and associated $p$-values for Kruskal Wallis tests (two-tailed; significance level: 0.05).

\begin{tabular}{|c|c|c|c|}
\hline \multicolumn{4}{|c|}{ Test F-M results Multiclass ANN } \\
\hline \multicolumn{2}{|c|}{ Feature ranking $C$ value } & \multicolumn{2}{|c|}{ Number of features, $N$} \\
\hline$C=0.1$ & $0.9795 \pm 0.0077$ & $N=5$ & $0.9737 \pm 0.0058$ \\
\hline$C=0.01$ & $0.9805 \pm 0.0054$ & $N=10$ & $0.9791 \pm 0.0057$ \\
\hline$C=0.001$ & $0.9790 \pm 0.0043$ & $N=15$ & $0.9828 \pm 0.0031$ \\
\hline$C=0.0001$ & $0.9796 \pm 0.0055$ & $N=20$ & $0.9770 \pm 0.0044$ \\
\hline$C=1 e^{-5}$ & $0.9785 \pm 0.0052$ & $N=25$ & $0.9760 \pm 0.0088$ \\
\hline$C=1 \mathrm{e}^{-6}$ & $0.9735 \pm 0.0069$ & $N=30$ & $0.9758 \pm 0.0092$ \\
\hline$C=5 e^{-7}$ & $0.9752 \pm 0.0104$ & $N=35$ & $0.9770 \pm 0.0078$ \\
\hline$C=1 \mathrm{e}^{-7}$ & $0.9785 \pm 0.0060$ & $N=39$ & $0.9789 \pm 0.0083$ \\
\hline$C=1 \mathrm{e}^{-8}$ & $0.9736 \pm 0.0094$ & & \\
\hline $\begin{array}{l}\text { p-value (Kruskal } \\
\text { Wallis) }\end{array}$ & 0.5262 & $\begin{array}{l}\text { p-value (Kruskal } \\
\text { Wallis) }\end{array}$ & 0.1437 \\
\hline
\end{tabular}

literature and similarly to our previous study [17]. However, significant differences were not found between performance measures (Acc and FM) regarding the training phase (Table 3 in SM).

In fact, the good performance of SVM classifier while dealing with this type of problems was already reported in our previous study [17]. Despite the fact that in this case a higher number of features is required to accurately differentiate noisy from APWs (between 15 and 20) in comparison with the latter study ( 5 features), being more computationally demanding, it has the advantages of, beyond being trained for 
Table 5

Results of the statistical tests performed in order to evaluate the best classifier in terms of performance values obtained both in the train and test (significance value: 0.05 ; Student $t$-test; two-tailed).

\begin{tabular}{|c|c|c|c|}
\hline Train & & Test & \\
\hline Accuracy (Acc) & F-Measure (F-M) & Accuracy (Acc) & F-Measure (F-M) \\
\hline 0.0806 & 0.0706 & $0.0011^{*}$ & $0.0004 *$ \\
\hline
\end{tabular}

* Statistically significant (significance level: 0.05).

distinguishing noisy from real APWs, it is also able to detect abnormal and normal APWs. This is possible for the cost of more 10 features, with a better performance. Furthermore, the selected set of the most suitable features has attributes in common with the best features set selected in the study of 2015, which reinforces their role in providing useful information about this type of signal [17].

The majority of the studies dedicated to APW, reported less expedite performances because their purpose was not to distinguish only between healthy and pathologic APWs, but rather on different number of APW types with different physiological relevance. This is the case of Joshi et al. [18] which proposed a method to differentiate between eight different types of pulse waves, using statistical metrics instead of machine learning algorithms, achieving limited performance results around 0.86 . Alty et al. [82], similarly with this present study applied both binary SVM and ANN in order to automatically diagnose arterial stiffness through APW evaluation, by distinguishing healthy from pathologic waveforms. Despite the fact that the population evaluated in their study was approximately the double in number than the present study, they reported performance values which, in general, did not achieved 0.93 for both SVM and ANN, including more computationally demanding features.

The most significant limitation of the present study rely on the population sample size, which, despite being equivalent with several studies found in literature, if larger, higher could be the generalization degree of the classification method proposed here. Additionally, our conclusions must be further validated using an APW dataset including patient's information from other clinical centres.

\section{Conclusions}

With the development of novel solutions to measure vital signals, an exhaustive study of the features that characterize those signals and their correlations is imposed. This paper describes a study of arterial pulse waveform-derived features, for distinguishing normal from pathological and noise segments.

The comparison between two classifiers, SVM and NN, for the signal type automatic classification allowed reassert the higher performance of SVM that represents here the best solution. SVM performs significantly better to distinguish between "noisy" from "pathological" and "non-pathological" APWs, achieving maximal F-Measure and Accuracy values of 0.9957 and 0.9956 , respectively, considering an 823 training versus 225 test samples evaluation scheme.

The order by which the features are chosen by an original set (morphologic, time domain statistics, cross-correlation and wavelet features) did not show to be significantly different for the classifier test performance in this type of problem. The results obtained here showed the potential of the proposed method in distinguishing those three important clinical outcomes (healthy, pathologic and noise), due to its simplicity, low complexity in comparison with the existent literature algorithms, its near- $100 \%$ test performance, and its ability to remove noisy signals which could lead to misdiagnoses.

\section{Author contribution}

TP acquired the signals, JP and TP developed and performed the computational calculus, JP, JC and TP analysed the results. All authors contribute to write the various iterations of the paper and approved the final manuscript.

\section{Competing interests}

The authors declare no conflict of interest.

\section{Summary Points}

- Development of an automatic method to distinguish normal to the pathological arterial pulse pressure waveforms (APW) from non-relevant information (noisy segments) acquired by a novel optical system during a clinical examination.

- The APW dataset analysed was composed by signals acquired in a clinical environment from a total of 213 subjects, including healthy volunteers and non-healthy patients.

- The signals were parameterised by means of 39 pulse features: morphologic, time domain statistics, cross-correlation and wavelet-derived features.

- The multiclass Support Vector Machine Recursive Feature Elimination (SVM RFE) method was used to select the most relevant features.

- A comparative study was performed in order to evaluate the performance of two types of classifiers: Support Vector Machine (SVM) and Artificial Neuronal Network (ANN).

- SVM achieved a statistically significant better performance.

\section{Acknowledgments}

Authors acknowledge the support from Fundação para a Ciência e a Tecnologia for funding (Grants with references SFRH/BD/79334/2011 and PD/BI/114377/2016). Project developed under the initiative of QREN, funding by UE/FEDER, through COMPETE.

\section{Appendix A. Supplementary data}

Supplementary data associated with this article can be found, in the online version, at http://dx.doi.org/10.1016/j.ijmedinf.2017.10.011.

\section{References}

[1] E.J. Kim, C.G Park, J.S. Park, S.Y. Suh, C.U. Choi, J.W. Kim, S.H. Kim, H.E. Lim, S.W. Rha, H.S. Seo, D.J. Oh, Relationship between blood pressure parameters and pulse wave velocity in normotensive and hypertensive subjects: invasive study, J. Hum. Hypertens. 21 (no. 2) (2007) 141-148.

[2] H.T. Ma, A blood pressure monitoring method for stroke management, Biomed Res. Int. vol 2014, (2014) 7.

[3] A.P. Avolio, M. Butlin, A. Walsh, Arterial blood pressure measurement and pulse wave analysis? their role in enhancing cardiovascular assessment, Physiol. Meas. vol. 31, (no. 1) (2010) 1-47.

[4] H. Tanaka, F.A. Dinenno, K.D. Monahan, C.M. Clevenger, C.A. DeSouza, D.R. Seals, Aging, habitual exercise, and dynamic arterial compliance, Circulation vol. 102, (no. 11) (2000) 1270-1275.

[5] R. Klocke, J.R. Cockcroft, G.J. Taylor, I.R. Hall, D.R. Blake, Arterial stiffness and central blood pressure, as determined by pulse wave analysis, in rheumatoid arthritis, Ann. Rheum. Dis. 62 (no. 5) (2003) 414-418.

[6] M. Crilly, C. Coch, M. Bruce, H. Clark, D. Williams, Indices of cardiovascular function derived from peripheral pulse wave analysis using radial applanation tonometry: a measurement repeatability study, Vasc. Med. 12 (no. 3) (2007) $189-197$.

[7] J. Lee, R.G. Mark, An investigation of patterns in hemodynamic data indicative of impending hypotension in intensive care, Biomed. Eng. Online vol. 9, (January (1)) (2010).

[8] K. Hirata, T. Yaginuma, M.F. O'Rourke, M. Kawakami, Age-related changes in carotid artery flow and pressure pulses: possible implications for cerebral 
microvascular disease, Stroke 37 (no. 10) (2006) 2552-2556.

[9] N.A. Shirwany, M. Zou, Arterial stiffness: a brief review, Acta Pharmacol. Sin. 31 (no. 10) (2010) 1267-1276.

[10] G.E. McVeigh, C.W. Bratteli, D.J. Morgan, C.M. Alinder, S.P. Glasser, S.M. Finkelstein, J.N. Cohn, Age-related abnormalities in arterial compliance identified by pressure pulse contour analysis: aging and arterial compliance, Hypertension 33 (no. 6) (1999) 1392-1398.

[11] T. Pereira, C. Correia, J. Cardoso, Novel methods for pulse wave velocity measurement, J. Med. Biol. Eng. 35 (no. 5) (2015) 555-565.

[12] T. Pereira, I. Santos, T. Oliveira, P. Vaz, T. Correia, T. Pereira, H. Santos, H. Pereira, V. Almeida, J. Cardoso, C. Correia, Characterization of optical system for hemodynamic multi-Parameter assessment, Cardiovasc. Eng. Technol. 4 (no. 1) (2013) 87-97.

[13] T. Pereira, I. Santos, T. Oliveira, P. Vaz, H. Santos, H. Pereira, V. Almeida, J. Cardoso, Local PWV and other hemodynamic parameters assessment: validation of a new optical technique in an healthy population, Barcelona, Spain, BIOSIGNALS 2013-6th International Conference on Bio-inspired Systems and Signal Processing, vol. 1, 2013, pp. 61-69.

[14] T. Pereira, I. Santos, T. Oliveira, P. Vaz, T. Pereira, H. Santos, H. Pereira, C. Correia, J. Cardoso, Pulse pressure waveform estimation using distension profiling with contactless optical probe, Med. Eng. Phys. (2014) 1515-1520.

[15] T. Pereira, T. Pereira, H. Santos, H. Pereira, C. Correia, J. Cardoso, Assessment of carotid distention waveform and local pulse wave velocity determination by a novel optical system, Artery Res. vol. 8, (no. 4) (2014) 135.

[16] T. Pereira, T.S. Pereira, H. Santos, C. Correia, J. Cardoso, Arterial pulse pressure waveform monitoring by novel optical probe, Int. J. Cardiol. vol 179, (2015).

[17] T. Pereira, J.S. Paiva, C. Correia, J. Cardoso, An automatic method for arterial pulse waveform recognition using KNN and SVM classifiers, Med. Biol. Eng. Comput. 54 (no. 7) (2016) 1049-1059.

[18] R.R. Joshi, Automatic detection of pulse morphology patterns \& amp; cardiac risks, J. Biomed. Sci. Eng. 5 (no. 6) (2012) 315-322.

[19] W. Zuo, D. Zhang, D. Zhang, H. Zhang, N. Li, Classification of pulse waveforms using edit distance with real penalty, EURASIP J. Adv. Signal Process. vol. 2010, (2010).

[20] D. Jia, D. Zhang, N. Li, Pulse waveform classification using support vector machine with gaussian time warp edit distance kernel, Comput. Math. Methods Med. vol 2014, (2014).

[21] Y.Z. Mehran, New application of wavelet transform in classification the arterial pulse signals, ICOSSE'06 Proceedings of the 5th WSEAS International Conference on System Science and Simulation in Engineering (2006) 448-453.

[22] R.J. Byrd, S.R. Steinhubl, J. Sun, S. Ebadollahi, W.F. Stewart, Automatic identification of heart failure diagnostic criteria, using text analysis of clinical notes from electronic health records, Int. J. Med. Inform. 83 (no. 12) (2014) 983-992.

[23] T. Zheng, W. Xie, L. Xu, X. He, Y. Zhang, M. You, G. Yang, Y. Chen, A machine learning-based framework to identify type 2 diabetes through electronic health records, Int. J. Med. Inform. 97 (2017) 120-127.

[24] R. Prashanth, S. Dutta Roy, P.K. Mandal, S. Ghosh, High-Accuracy detection of early parkinson's disease through multimodal features and machine learning, Int. J. Med. Inform. 90 (2016) 13-21.

[25] E. Hemphill, J. Lindsay, C. Lee, I.I. Măndoiu, C.E. Nelson, Feature selection and classifier performance on diverse biological datasets, BMC Bioinf. vol. 15, (Suppl 1, no. 13) (2014) S4.

[26] X. Zhou, D.P. Tuck, MSVM-RFE: Extensions of SVM-RFE for multiclass gene selection on DNA microarray data, Bioinformatics 23 (no. 9) (2007) 1106-1114.

[27] O.Y. Atkov, S.G. Gorokhova, A.G. Sboev, E.V. Generozov, E.V. Muraseyeva, S.Y. Moroshkina, N.N. Cherniy, Coronary heart disease diagnosis by artificial neural networks including genetic polymorphisms and clinical parameters, J. Cardiol. 59 (no. 2) (2012) 190-194.

[28] K.U. Rani, Analysis of heart diseases dataset using neural network approach, Int. J. Data Min. Knowl. Manag. Process 1 (no. 5) (2011) 1-8.

[29] E.O. Olaniyi, O.K. Oyedotun, K. Adnan, Heart diseases diagnosis using neural networks arbitration, Int. J. Intell. Syst. Appl. 7 (no. 12) (2015) 75-82.

[30] A. De Gaetano, S. Panunzi, F. Rinaldi, A. Risi, M. Sciandrone, A patient adaptable ECG beat classifier based on neural networks, Appl. Math. Comput. vol. 213, (no. 1) (2009) 243-249.

[31] S.H. El-Khafif, M.A. El-Brawany, Artificial neural network-Based automated ECG signal classifier, ISRN Biomed. Eng. vol 2013, (2013) 3-6.

[32] S. Poungponsri, X.-H. Yu, An adaptive filtering approach for electrocardiogram (ECG) signal noise reduction using neural networks, Neurocomputing 117 (2013) 206-213.

[33] A.A.M. Khalaf, M.M. Ibrahim, H.F.A. Hamed, Performance study of adaptive filtering and noise cancellation of artifacts in ECG signals 2015, 17th Int. Conf. Adv. Commun. Technol. vol. 2015, (2015) 394-401 (Augus, no. July).

[34] V.J. Ribas Ripoll, A. Wojdel, E. Romero, P. Ramos, J. Brugada, ECG assessment based on neural networks with pretraining, Appl. Soft Comput. 49 (2016) 399-406.

[35] H. Lee, S.-Y. Shin, M. Seo, G.-B. Nam, S. Joo, Prediction of ventricular tachycardia one hour before occurrence using artificial neural networks, Sci. Rep vol. 6, (no. April) (2016) 32390.

[36] A. Orjuela-Cañón, H. Posada-Quintero, D. Delisle-Rodríguez, M. Cuadra-Sanz, R. Fernández de la Vara-Prieto, A. López-Delis, Onset and peak detection over pulse wave using supervised SOM network, Int. J. Biosci. Biochem. Bioinforma. 3 (no. 2) (2013) 133-137.

[37] D. Quinn, M. Kinsley, T. Whitaker, J. Seller, Systems and Methods for Measuring Arterial Stiffness, (2012).

[38] A.-D. Almási, V. Woźniak, Y. Leblebici, T. Engbersen, Review of advances in neural networks: neural design technology stack, Proc. ELM-2014, Vol. 1 SE - 31 vol. 3,
(2015) 367-376.

[39] F. Salih, O. Abdallah, Q. Qananwah, A. Bolz, Normalized area under catacrotic phase of the photoplethysmogram pulse for estimating vascular aging, J. Biomed. Eng. vol. 9, (2012).

[40] T. Pereira, I. Santos, T. Oliveira, P. Vaz, T. Pereira, H. Santos, H. Pereira, C. Correia, J. Cardoso, Pulse pressure waveform estimation using distension profiling with contactless optical probe, Med. Eng. Phys. 36 (no. 11) (2014) 1515-1520.

[41] D. Sueta, E. Yamamoto, T. Tanaka, Y. Hirata, K. Sakamoto, K. Tsujita, S. Kojima, K. Nishiyama, K. Kaikita, S. Hokimoto, H. Jinnouchi, H. Ogawa, Association of estimated central blood pressure measured non-invasively with pulse wave velocity in patients with coronary artery disease, IJC Hear. Vasc. vol. 8, (2015) 52-54.

[42] M.L. Meyer, H. Tanaka, P. Palta, M.D. Patel, R. Camplain, D. Couper, S. Cheng, A. Al Qunaibet, A.K. Poon, G. Heiss, Repeatability of central and peripheral pulse wave velocity measures: the atherosclerosis risk in communities (ARIC) study, Am. J. Hypertens. (2015) 470-475.

[43] J. Doupis, N. Papanas, A. Cohen, L. McFarlan, E. Horton, Pulse wave analysis by applanation tonometry for the measurement of arterial stiffness, open cardiovasc, Med. J. 10 (no. 1) (2016) 188-195.

[44] A. Cozma, D. Leucuţa, D. Pop, D. Tudor, Impact of the defining criteria and components of metabolic syndrome on arterial stiffness parameters, Appl. Med. Informatics 27 (no. 4) (2010) 39-46.

[45] I. Mandal, N. Sairam, Accurate prediction of coronary artery disease using reliable diagnosis system, J. Med. Syst. 36 (no. 5) (2012) 3353-3373.

[46] S. Karpagachelvi, M. Arthanari, M. Sivakumar, ECG feature extraction techniques a survey approach, Int. J. Comput. Sci. Inf. Secur. 8 (no. 1) (2010) 76-80.

[47] M. Kasprowicz, S. Asgari, M. Bergsneider, M. Czosnyka, R. Hamilton, X. Hu, Pattern recognition of overnight intracranial pressure slow waves using morphological features of intracranial pressure pulse, J. Neurosci. Methods 190 (no. 2) (2010) 310-318.

[48] G.S. Nayak, Classification of ECG signals using ANN with resilient back propagation algorithm, Int. J. Comput. Appl. vol. 54, (no. 6) (2012) 20-23.

[49] P.F. Diez, V. Mut, E. Laciar, A. Torres, E. Avila, Application of the empirical mode decomposition to the extraction of features from EEG signals for mental task classification, Conf. Proc. Annu. Int. Conf. IEEE Eng. Med. Biol. Soc. IEEE Eng. Med. Biol. Soc. Conf. vol 2009, (2009) 2579-2582.

[50] T. Weber, J. Auer, M.F. O'Rourke, E. Kvas, E. Lassnig, R. Berent, B. Eber, Arterial stiffness, wave reflections, and the risk of coronary artery disease, Circulation vol. 109, (no. 2) (2004) 184-189.

[51] M. De Melis, U. Morbiducci, E.R. Rietzschel, M. De Buyzere, A. Qasem, L. Van Bortel, T. Claessens, F.M. Montevecchi, A. Avolio, P. Segers, Blood pressure waveform analysis by means of wavelet transform, Med. Biol. Eng. Comput. vol. 47, (no. 2) (2009) 165-173.

[52] J. Kips, F. Vanmolkot, D. Mahieu, S. Vermeersch, I. Fabry, J. de Hoon, L. Van Bortel, P. Segers, The use of diameter distension waveforms as an alternative for tonometric pressure to assess carotid blood pressure, Physiol. Meas. 31 (no. 4) (2010) 543-553.

[53] A. Pachauri, M. Bhuyan, Wavelet transform based arterial blood pressure waveform delineator, Int. J. Biol. Biomed. Eng. vol. 6, (no. 1) (2012).

[54] D. Álvarez, R. Hornero, J.V. Marcos, F. Del Campo, Feature selection from nocturnal oximetry using genetic algorithms to assist in obstructive sleep apnoea diagnosis, Med. Eng. Phys. 34 (no. 8) (2012) 1049-1057.

[55] W. He, S. Li, H. Xiao, C. Yu, H. Lin, An arterial elasticity index algorithm based on wavelet transform and curve fitting, J. Inf. Comput. Sci. 9 (no. 12) (2012) 3379-3389.

[56] J.B. Janney, S. Sruthi P, Dicrotic notch detection and analysis of arterial pulse by using discrete wavelet, OSIET J. Commun. Electron. vol. 4, (no. May) (2012) 2012.

[57] M.H. Nguyen, F. de l aTorre, Optimal feature selection for support vector machines, Pattern Recognit. 43 (no. 3) (2010) 584-591.

[58] T.M. Huang, V. Kecman, Gene extraction for cancer diagnosis by support vector machines an improvement and comparison with nearest, Artif. Intell. Med. vol. 35, (no. 1) (2005) 185-194.

[59] X. Hu, T. Glenn, F. Scalzo, M. Bergsneider, C. Sarkiss, N. Martin, P. Vespa, Intracranial pressure pulse morphological features improved detection of decreased cerebral blood flow, Physiol. Meas. 31 (no. 5) (2010) 679-695.

[60] I. Guyon, A. Elisseeff, An introduction to variable and feature selection, J. Mach. Learn. Res. 3 (no. 3) (2003) 1157-1182.

[61] Y. Liu, Y.F. Zheng, FS_SFS: A novel feature selection method for support vector machines, Pattern Recognit. 39 (no. 7) (2006) 1333-1345.

[62] X. Wang, J. Tian, A gene selection method for cancer classification, Comput. Math. Methods Med. vol 2012, (2012) 586246.

[63] K.B. Duan, J.C. Rajapakse, H. Wang, F. Azuaje, Multiple SVM-RFE for gene selection in cancer classification with expression data, IEEE Trans. Nanobioscience 4 (no. 3) (2005) 228-233.

[64] V. Guyon, Isabelle, Jason Weston, Stephen Barnhill, Vapnik, Gene selection for cancer classification using support vector machines, Mach. Learn. 46 (no. 4) (2002) 389-422.

[65] F. Gargiulo, A. Fratini, M. Sansone, C. Sansone, Subject identification via ECG fiducial-based systems: influence of the type of QT interval correction, Comput. Methods Programs Biomed. 121 (no. 3) (2015) 127-136.

[66] J.P. Marques, Pattern Recognition: Concepts, Methods, and Applications, 1 st Ed, Springer Verlag, 2001.

[67] S.H.eA.A.M. Hejazi, S. Al-Haddad, Y. Singh, Multiclass support vector machines for classification of ECG data with missing values, Appl. Artif. Intell. 29 (2015) $660-674$.

[68] X. Wang, J. Tian, A gene selection method for cancer classification, Comput. Math. Methods Med. vol. 2012, (2012). 
[69] A. Wilin, Gene selection for cancer classification using support vector machines, Mach. Learn. (no. 46) (2002) 389-422.

[70] M.I. Schlesinger, V. Hlavac, Statistical Pattern Recognition Toolbox, (2017) ([Online]. Available: http://cmp.felk.cvut.cz/cmp/software/stprtool /).

[71] P. Rani, C. Liu, N. Sarkar, E. Vanman, An empirical study of machine learning techniques for affect recognition in human-robot interaction, Pattern Anal Appl. 9 (no. 1) (2006) 58-69.

[72] D.G.S. Richard, O. Duda, Peter E. Hart, Pattern Classification, John Wiley \& Sons, 2012.

[73] S. Afifi, H. Gholam Hosseini, R. Sinha, Hardware acceleration of SVM-based classifier for melanoma images, Lect. Notes Comput. Sci 9555 (2016) 235-245 (including Subser. Lect. Notes Artif. Intell. Lect. Notes Bioinformatics).

[74] L.W.S. Cogill, Support vector machine model of developmental brain gene expression data for prioritization of Autism risk gene candidates, Bioinformatics 32 (no. 23) (2016).

[75] N. Mammadova, I. Keskin, Application of the support vector machine to predict subclinical mastitis in dairy cattle, ScientificWorldJournal vol. 2013, (2013) 603897.

[76] B.C, H.S.D. Debatosh Guha, Arrhythmia classification by nonlinear kernel-based ECG signal modeling, Comput. Commun. Electr. Technol. (2017) 325-330.

[77] G.E. Sakr, M. Mokbel, A. Darwich, Comparing deep learning and support vector machines for autonomous waste sorting, in Multidisciplinary Conference on Engineering Technology (IMCET), IEEE Int. (2016) 207-212.
[78] A.-H.M. Jallad, L.B. Mohammed, Hardware support vector machine (SVM) for satellite on-board applications, Adaptive Hardware and Systems (AHS) 2014 NASA/ ESA Conference on (2014).

[79] T. Jeon, B. Kim, M. Jeon, B.-G. Lee, Implementation of a portable device for realtime ECG signal analysis, Biomed. Eng. Online vol. 13, (2014) 160.

[80] T. Pereira, J.S. Paiva, C. Correia, J. Cardoso, An automatic method for arterial pulse waveform recognition using KNN and SVM classifiers, Med. Biol. Eng. Comput. (2015) 1049-1059.

[81] F. Melgani, Y. Bazi, Classification of electrocardiogram signals with support vector machines and particle swarm optimization, IEEE Trans. Inf. Technol. Biomed. 12 (no. 5) (2008) 667-677.

[82] S.R. Alty, N. Angarita-jaimes, S.C. Millasseau, P.J. Chowienczyk, Predicting arterial stiffness from the digital volume pulse waveform, Biomed. Eng. IEEE Trans. 54 (no. 12) (2007) 2268-2275.

[83] T. Frontzek, T.N. Lal, R. Eckmiller, Predicting the nonlinear dynamics of biological neurons using support vector machines with different kernels, Neural Networks, 2001. Proceedings. IJCNN '01. International Joint Conference on (2001) 1492-1497.

[84] S.B. Kotsiantis, Supervised machine learning: a review of classification techniques, Informatica 31 (2007) 249-268.

[85] B. Yegnanarayana, Artificial Neural Networks, (2009).

[86] D. Svozil, V. Kvasnička, J. Pospíchal, Introduction to multi-layer feed-forward neural networks, Chemom. Intell. Lab. Syst. 39 (no. 1) (1997) 43-62. 